\title{
Inhibition of the ruminal bacterium Ruminococcus flavefaciens by ruminal fungi
}

\author{
KN Joblin, GE Naylor \\ Grassiands Research Centre, AgResearch, Private Bag 11008, \\ Palmerston North, New Zealand.
}

\begin{abstract}
Grazing ruminants depend upon fibrolytic bacteria and fungi to digest forage fibre in the rumen. Little is known about interactions between the fibrolytic populations. Ruminal fungi are potent degraders of lignocellulosic tissues and are believed to have an important role in fibre digestion in the rumen (Orpin and Joblin, 1988, in: The Rumen Microbial Ecosystem, Hobson, ed, Elsevier, London, 129-150). Ruminococcus flavefaciens is one of three major plant cell-wall degrading bacteria in the rumen (Stewart and Bryant, 1988, in: The Rumen Microbial Ecosystem, Hobson, ed, Elsevier, London, 21-75). R. flavefaciens has been shown to inhibit cellulolysis by the fungus Neocallimastix frontalis (Bernalier et al, 1993, J Gen Microbiol, 139, 873-880; Stewart et al, 1992, FEMS Microbiol Lett, 97, 83-88) but had no marked effect on xylanolysis by the fungus Piromyces communis (Williams et al, 1991, Lett Appl Microbiol, 12, 232-235). We report here results showing that extra-cellular compounds produced by ruminal fungi inhibit $R$. flavefaciens growing on xylan.
\end{abstract}

R. flavefaciens FD1 was grown on xylan in coculture with 3 different ruminal fungi. Xylan utilization in all cocultures were similar to that of the R. flavefaciens growing axenically. However, fermentation product profiles from all the cocultures were very different to that of the $R$. flavefaciens. The product profiles were similar to those of the respective fungi (two Piromyces sp. and one Neocallimastix sp.) growing in monoculture. This showed that fungi dominated $R$. flavefaciens in the cocultures and suggested that fungi were actively inhibiting the bacterium.

To test this, inhibition experiments were carried out using fungal supernatants filtered through a 0.45 filter. Cell-free supernatant prepared from Piromyces communis $\mathrm{B} 19$ strongly inhibited xylanolysis by $R$. flavefaciens. Autoclaved fungal supernatants were not inhibitory. In contrast, the fungal supernatant had no effect on xylanolysis by Ruminococcus albus. Growth of $R$. flavefaciens on xylan was inhibited but growth on soluble sugars was not. We have found no evidence for bacteriocin-like activity similar to that found when $R$. albus inhibits $R$. flavefaciens (Odenyo et al, 1994, Appl Environ Microbiol, 60, 36883696). Tests using agar plates have yet to show any effect from the inhibitor on xylanclearing by $R$. flavefaciens supernatant. 